\title{
Médiévales
}

Langues, Textes, Histoire

\section{Le royaume lombard et les duchés : formes et moyens d'une intégration progressive}

The Kingdom of Lombardy and the Duchies: Forms and Methods of a Progressive Integration.

\section{Yves-Mary Verhoeve}

\section{OpenEdition}

\section{Journals}

Édition électronique

URL : https://journals.openedition.org/medievales/1570

DOI : $10.4000 /$ medievales. 1570

ISSN : 1777-5892

Éditeur

Presses universitaires de Vincennes

Édition imprimée

Date de publication : 1 décembre 2006

Pagination : 21-36

ISBN : 978-2-84292-193-4

ISSN : 0751-2708

\section{Référence électronique}

Yves-Mary Verhoeve, «Le royaume lombard et les duchés : formes et moyens d'une intégration progressive », Médiévales [En ligne], 51 | automne 2006, mis en ligne le 27 mars 2009, consulté le 23 avril 2022. URL : http://journals.openedition.org/medievales/1570 ; DOI : https://doi.org/10.4000/

medievales. 1570

Ce document a été généré automatiquement le 23 avril 2022

Tous droits réservés 


\section{Le royaume lombard et les duchés : formes et moyens d'une intégration progressive}

The Kingdom of Lombardy and the Duchies: Forms and Methods of a Progressive Integration.

Yves-Mary Verhoeve

\section{NOTE DE L'ÉDITEUR}

Les généalogies accompagnant cet article sont consultables dans la version imprimée du numéro 51.

Au bout de dix ans de pouvoir ducal, les Lombards se décidèrent finalement, d'un commun accord, à prendre un roi : Authari, fils du prince Cleph évoqué plus haut. Comme signe de sa dignité, ils le nommèrent Flavius, un prénom que tous ceux qui furent par la suite rois des Lombards ont porté avec bonheur. Sous son règne, les ducs du moment assignèrent aux besoins de la cour la moitié de leurs biens pour l'objectif de reconstruction de la royauté, afin de dégager de quoi subvenir au roi lui-même, à son entourage et à ceux qui étaient à son service dans les différents emplois. Les populations en revanche, soumises au tribut, furent réparties entre les hôtes lombards. Le royaume offrait à vrai dire ceci de surprenant qu'il n'existait nulle violence, qu'il ne s'y machinait aucune trahison; personne n'extorquait personne, nul ne pillait; aucun vol, aucun brigandage : chacun vaquait sans crainte où bon lui semblait, en toute sécurité ${ }^{1}$.

L'élection d'Authari en 584 intervient après dix ans d'interrègne, où le pouvoir en Italie avait été exercé par les ducs lombards. Cependant, la menace franque et byzantine pesait lourdement sur les positions lombardes. Les duchés, autonomes, sans réelle cohésion entre eux risquaient d'être absorbés par leurs puissants voisins. C'est dans ce contexte qu'il faut envisager la restauration de la royauté lombarde en 584. 
2 Les ducs, installés dans les cités, ont contribué à accroître la domination lombarde sur l'Italie et à pérenniser l'entreprise entamée par le roi Alboin en 568. Toutefois, l'absence de pouvoir centralisateur se révéla très vite préjudiciable dans les luttes qui les opposaient aux Byzantins et aux Francs. Ces derniers multipliaient les expéditions militaires et leur alliance risquait de remettre en cause la domination lombarde, d'autant que certains ducs, achetés par les Byzantins, avaient déjà fait défection pour se soumettre à l'autorité de l'empereur ${ }^{2}$. Cependant, la présentation que fait Paul Diacre des événements laisse songeur. En effet, la soumission volontaire des duchés à l'autorité du roi n'allait pas de soi. La constitution du royaume lombard est le fruit d'un processus lent, dans lequel l'opposition entre le roi et certains duchés constitue une composante essentielle. Les ducs sont dans une large mesure restés jaloux de leur autonomie et n'ont eu de cesse de la défendre face au pouvoir centralisateur des souverains lombards. La constitution du royaume lombard est l'histoire d'une intégration progressive des duchés. Cette histoire n'est pas linéaire, elle est faite de nombreuses réactions contre le pouvoir royal. Ainsi, il importe de s'attacher, d'une part, aux moyens mis en œuvre par les souverains lombards pour soumettre ces turbulents duchés, et d'autre part aux réactions de ces derniers face à la centralisation croissante du pouvoir.

3 Les limites chronologiques du sujet se sont imposées naturellement au regard des sources. La connaissance des relations entre le roi et les ducs lombards est largement conditionnée par l'œuvre de Paul Diacre, qui, même si elle doit être maniée avec précaution, offre de précieux témoignages et un récit événementiel assez complet. À ce titre, j'ai choisi de prendre comme point de départ l'année 584, date de l'élection d'Authari, et l'année 744, la mort de Liutprand, comme borne finale. Ce choix s'explique par le caractère lacunaire des sources susceptibles de présenter un récit des événements après la mort de Liutprand. De plus, le règne de Liutprand constitue dans une large mesure l'apogée du royaume lombard, tant sur le plan territorial, que sur celui de la centralisation du pouvoir, avec Pavie comme capitale ${ }^{3}$. De fait, si l'on excepte les cas des duchés du sud, Spolète et Bénévent, qui n'appartiennent pas stricto sensu au regnum, le processus d'intégration des duchés est consommé à la fin de ce règne. Il faut toutefois remarquer que pour ces deux duchés la situation est ambiguë. Ils semblent au départ relativement autonomes jusqu'au milieu du vile siècle, mais par la suite étroitement associés au regnum ${ }^{4}$.

Dans un premier temps, le roi lombard est choisi parmi les ducs avant que ne s'impose une continuité dynastique, avec la descendance de Théodelinde ou de son frère Gundoald. Il est évident que cette élection n'allait pas de soi, comme tout enjeu de pouvoir, plusieurs compétiteurs devaient se présenter. L'élection marginalisait les compétiteurs évincés et suscitait rancœurs et rivalités ${ }^{5}$. De fait, il convient d'envisager, les appuis du roi lors de son élection, les réseaux de soutiens et de contestations de la royauté durant le règne, et parallèlement la manière dont celle-ci s'affermit progressivement, notamment par l'instauration d'une continuité dynastique. À ce titre, les stratégies matrimoniales des rois lombards avec les duchés constituent un aspect éclairant sur la question. En effet, le mariage d'un membre de la famille royale avec un duc turbulent, de même que la désignation d'un homme de la parentèle royale à la tête d'un duché constituent sur toute la période l'un des moyens privilégiés de l'intégration des duchés dans la sphère d'influence du regnum. 
Les premiers tâtonnements (584-636), de l'élection d'Authari à celle de RothariL'élection du roi : un choix stratégique

Lors de la restauration de la royauté lombarde, le choix des ducs se porte sur Authari, le fils de Cleph (572-574), c'est-à-dire le dernier souverain lombard. Toutefois, c'est davantage son appartenance à une prestigieuse famille ${ }^{6}$ et le fait qu'il soit l'un des leurs qui doit retenir l'attention. En effet, même s'il est impossible de déterminer la cité à la tête de laquelle se trouvait Authari, nous savons par le pseudo-Frédégaire que celui-ci était un duc ${ }^{7}$.

6 L'élection d'Authari (584-590) intervient dans un contexte de recul de la domination lombarde, battue en brèche par les Francs. En effet, l'absence d'un centre politique et d'un commandement militaire centralisé se révèle vite préjudiciable pour faire face aux incursions franques en Italie, qui se multiplient ${ }^{8}$. De plus, en 583 ou 584 , l'empereur byzantin Maurice obtient de Childebert II, le roi d'Austrasie, en échange de 50000 sous d'or, une intervention armée des Francs en territoire lombard ${ }^{9}$. Certes, celle-ci se solde par un échec, mais elle a obligé les ducs lombards et leur population à se retrancher derrière les murs de leurs cités, incapables de répondre à la menace et contraints à la négociation. Cet épisode révèle la faiblesse d'une domination lombarde reposant sur la superposition de commandements militaires éclatés, souvent réduits au territoire d'une cité. Ainsi, c'est sous la menace conjuguée des Francs et des Byzantins que s'impose la nécessité de restaurer la royauté, et c'est dans la même perspective qu'il faut interpréter le mariage d'Authari avec la bavaroise Théodelinde. Celle-ci est la fille du duc des Bavarois, Garibald et de la Lombarde Walderade (veuve de Théodebald I). La chronique du pseudo-Frédégaire la dit d'origine franque (ex genere Francorum). Les Bavarois, à cette époque, sont dans une large mesure dans la sphère d'influence franque ${ }^{10}$. Par ce mariage, il s'agissait donc de s'allier aux Francs ou tout au moins de neutraliser leur alliance avec les Byzantins.

Dans un premier temps, c'est donc à la lumière des relations avec l'extérieur, avec les Francs et les Byzantins, qu'il faut envisager les rapports entre le pouvoir royal et les duchés. À ce titre, le choix du roi des Lombards parmi les ducs est révélateur des menaces qui pèsent sur le royaume. Ainsi, à la mort d'Authari, le duc de Turin Agilulf est élevé à la royauté. Les conditions dans lesquelles s'effectue cette élection méritent d'être évoquées. En effet, à la mort d'Authari en 590, sa veuve, Théodelinde, après avoir pris conseil auprès des prudentes, désigne seule Agilulf comme époux et nouveau roi des Lombards ${ }^{11}$. Il est clair que les motifs politiques prédominent dans ce choix. De même, à la mort d'Agiluf (590-616), après un règne conjoint de Théodelinde et de leur fils Adaloald (616-626), c'est à nouveau le duc de Turin qui s'empare de la royauté : Arioald (626-636) ${ }^{12}$. Celui-ci détrône Théodelinde et son fils en 626, vraisemblablement en raison de leur politique trop ouvertement favorable aux Byzantins et aux Romains.

8 L'élection successive de deux ducs de Turin traduit bien les préoccupations des Lombards, et leurs préventions à l'égard de leurs voisins Francs. En effet, les années 588 et 590 sont marquées par une série d'incursions franques en territoire lombard ${ }^{13}$. La pression franque se faisant plus forte, le choix d'Agilulf et d'Arioald, tous deux ducs de Turin, est alors déterminant. Cette cité au nord de la plaine du Pô est un verrou important aux débouchés des passages alpins, que ce soient les cluses du Val de Suse et du Val d'Aoste, ou les cols comme celui du Mont Cenis ${ }^{14}$. Les ducs de Turin sont en contact direct avec leurs ennemis Francs, les connaissent et ont eu affaire à eux. C'est dans la même perspective, à un moment où la menace byzantine se fait plus pressante, 
qu'en 636, le duc de Brescia, Rothari (636-652) accède à la royauté ${ }^{15}$. Là encore, le choix de Brescia est significatif dans la mesure où cette cité se trouve aux marges des territoires Byzantins, et s'affirme comme l'une des bases de départ des incursions lombardes vers le sud, vers les territoires contrôlés par l'Empire. En somme, le roi est choisi parmi les ducs les plus au contact del'ennemi, peut-être les plus à même de connaître les interlocuteurs du camp adverse et ayant fait leur preuve face à eux.

S'imposer face aux ducs

9 L'attitude adoptée par les rois lombards à l'égard des ducs jusqu'au milieu du vil e siècle traduit également leurs préoccupations face aux Francs et aux Byzantins. En effet, le problème est triple. Il s'agit d'une part d'éliminer un certain nombre de ducs trop sensibles aux appels de Byzance, à l'instar des ducs de Brescello et de Pérouse, Droctulf et Maurice ${ }^{16}$. Ces deux ducs étaient passés du côté impérial, le premier lors de l'invasion franque de 583/4 et le second à la suite d'une offensive de l'exarque de Ravenne Romain (593/4). De fait, ces ducs tenaient leur cité au nom de l'empereur, ce qui menaçait directement la cohésion du regnum, en y maintenant des enclaves byzantines. Aussi, l'une des tâches des rois lombards a consisté à prendre les armes contre ces ducs, à assiéger leur cité pour finalement les supprimer. Le cas de Droctulf de Brescello est un bon exemple. En effet, Authari ne se contente pas de tuer le duc, il rase entièrement la cité, symbole du pouvoir rebelle et place convoitée par les Byzantins.

10 Le second problème des souverains lombards était d'écarter des ducs trop puissants, des compétiteurs possibles, susceptibles de remettre leur autorité en question. C'est par exemple le cas de Warnecausus à Pavie ${ }^{17}$. Paul Diacre nous dit que le roi Agilulf, après avoir conclu avec le roi des Francs Thierry II (595-613) une paix éternelle, élimine Zangrulf, duc de Vérone, Gaidulf, duc de Bergame et Warnecausus à Pavie. Ce dernier, même si l'Histoire des Lombards ne lui accorde pas de titre ducal, était selon toute vraisemblance duc de cette importante cité. Il est intéressant de noter que le roi Agilulf mâte simultanément deux révoltes, ayant lieu dans deux cités traditionnellement associées au pouvoir et à l'autorité des rois lombards : Vérone et Pavie. Vérone avait été aux premiers temps de la conquête, si ce n'est la capitale, du moins le centre de la domination lombarde, le roi Alboin y ayant élu domicile. De fait, la cité devait, à l'époque d'Agilulf, conserver un certain prestige ${ }^{18}$. De même, Pavie, constituait un nœud stratégique de la plaine du Pô, puisqu'elle contrôlait les voies fluviales vers Ravenne; depuis sa conquête par Alboin, elle bénéficiait non seulement d'une présence militaire importante, mais aussi d'un prestige incontesté auprès de l'ensemble des Lombards. Pavie était le cœur du royaume, ainsi que l'un des centres du pouvoir lombard, avec Milan notamment ${ }^{19}$. C'est à ce titre, qu'Agilulf, nouvellement installé sur le trône, s'empresse d'éliminer des ducs, certes rebelles à son autorité, mais surtout à la tête de cités dont le prestige est susceptible d'occulter la légitimité d'une fonction royale nouvellement acquise. Enfin, le roi semble aller plus loin dans la mesure où, dès ce moment, plus aucun duc n'est attesté à Pavie ${ }^{20}$, la cité s'affirmant progressivement durant les $\mathrm{VII}^{\mathrm{e}}$ et $\mathrm{VIII}^{\mathrm{e}}$ siècles comme l'unique capitale du royaume.

11 Le dernier problème pour le roi lombard consistait à stabiliser ses propres positions à l'intérieur du royaume, asseoir sa légitimité en se garantissant un réseau de soutiens. C'est dans ce cadre qu'intervient la mise en place d'un certain nombre de stratégies matrimoniales et de stratégies de parenté.

L'épouse du roi : une force légitimante du pouvoir royal 

leurs positions vis-à-vis des autres ducs en instaurant une forme de continuité dynastique, c'est-à-dire en se rattachant systématiquement à la lignée bavaroise issue de Théodelinde ${ }^{21}$. Une pratique courante consistait à épouser la veuve du roi défunt. Le nouveau souverain récupère et le prestige familial, et le prestige de son prédécesseur. Ainsi, Agilulf épouse Théodelinde à la mort d'Authari, Arioald épouse leur fille Gundeperge ${ }^{22}$, de même que Rothari ${ }^{23}$ et son fils Rodoald ${ }^{24}$ (652-653). Épouser la veuve du roi défunt revient à conférer au roi un surcroit de légitimité aux yeux des autres ducs et donc à asseoir sa position. Théodelinde et sa descendance vont constituer dans une certaine mesure la force légitimante du pouvoir royal. En effet, elle est la petitefille du roi Waccho (v. 510-v. 539). Or, ce dernier appartenait à la famille des Lithinges : «nom donné par les Lombards à l'une de leurs vieilles familles nobles ${ }^{25}$ ». Qui plus est Waccho était renommé parmi les guerriers, admiré pour sa bravoure et pour avoir soumis les Suèves. Épouser cette petite-fille de Lithinges, c'est se rattacher à cette famille, et qui plus est, conférer aux enfants mâles une prestigieuse ascendance. Ainsi, l'épouse, de par son origine, constitue un gage de légitimité pour l'époux, garantie d'une «reconnaissance sociale»; elle transmet le prestige de sa famille d'abord à l'époux, puis à sa progéniture. En cela, elle est le vecteur du «patrimoine spirituel » de la famille. Qui plus est, au fil des alliances, des mariages, ce patrimoine spirituel s'enrichit, il gagne en prestige. Ainsi, en épousant la fille de Garibald, les Lombards se sont liés à la prestigieuse dynastie des Agilolfing ${ }^{26}$. Théodelinde est dans une certaine mesure le résultat de la fusion de ces deux familles et, porte par là un "trésor virtuel » très convoité. Elle est le point de rencontre des Lithinges et des Agilolfing, et représente la synthèse de ces deux familles, désormais liées par le sang. Ce «trésor virtuel » explique l'attrait qu'a pu susciter, jusqu'au vIII ${ }^{\mathrm{e}}$ siècle la descendance de Théodelinde et la volonté systématique des souverains Lombards de se rattacher aux Agilolfing. La reine est ainsi le vecteur d'une mémoire familiale, mais également de la mémoire conjugale ${ }^{27}$.

S'assurer du soutien des duchés clés

Les rois vont également tenter d'imposer les membres de leur parenté à la tête de duchés clés, ou à l'inverse de faire entrer dans leur parenté ces ducs. Ainsi, le frère de Théodelinde, Gundoald, est placé à la tête du duché d'Asti ${ }^{28}$. Paul Diacre mentionne pour la première fois Gundoald, à l'occasion d'une incursion franque sur le territoire des Bavarois en 589. Cette attaque contraint Théodelinde et Gundoald à se réfugier en Italie. Théodelinde à l'issue de sa fuite retrouve Authari, à qui elle avait été fiancée, et l'épouse ${ }^{29}$. Gundoald n'a alors aucune fonction, aucun statut à la cour lombarde, si ce n'est celui de frère de la reine. Or, à sa mort vers 612, Paul Diacre le présente comme duc d'Asti, une cité qui était jusqu'alors dépendante du duché de Turin. Ainsi, d'autorité le roi, Authari ou Agilulf, a désigné le duc de cette cité (au sud de Turin) en contact avec le monde franc. Ce duché est donc une création ex novo de la part du roi ${ }^{30}$ et témoigne de la volonté de renforcer le dispositif de protection des «frontières » du royaume, et peut-être de contrôler par un membre de la parentèle royale le duché de Turin. De même, le beau frère d'Authari et d'Agilulf, Evin/Eoin ${ }^{31}$, se retrouve à la tête du duché de Trente ${ }^{32}$. À plusieurs reprises, il s'est illustré dans la défense de sa cité et du royaume contre les Francs. Vers 580, il repousse et tue le duc des Francs Cramnichis, conservant ainsi le Trentin aux mains des Lombards ${ }^{33}$. De plus, en 590, le roi Authari envoie une armée en Istrie, qu'il confie au duc Evin ${ }^{34}$. Pour les deux hommes, Gundoald et Evin, il s'agissait de sécuriser le nord du royaume, c'est-à-dire la frontière avec les 
Francs et les Bavarois, leurs alliés. Ces ducs, de par les mariages qu'ils avaient contractés, étaient intimement liés au roi. Leur position géographique, leur connaissance de leurs voisins francs les rendaient à même de garantir la paix au nord du royaume. C'est d'ailleurs à ce titre que le duc Evin de Trente est envoyé par deux fois pour négocier la paix avec les Francs en tant qu'ambassadeur ${ }^{35}$. La région du Trentin était hautement stratégique dans le cadre des relations avec les Francs, notamment la protection des cols alpins. Il importait donc d'y placer des hommes sûrs. Il ne semble pas que les désignations des ducs, effectuées d'autorité par le roi, aient suscité d'importantes réticences. En tous les cas, les sources n'en font pas état. Le caractère encore récent de la conquête au début $\mathrm{du} \mathrm{VII}^{\mathrm{e}}$ siècle explique le faible ancrage des politiques locales de transmission du pouvoir. Ceci est d'autant plus vrai pour les duchés créés de toutes pièces, comme celui d'Asti. Toutefois, dès le milieu du VII siècle ces stratégies de nomination et de substitution des ducs par le pouvoir royal, suscitent de nombreuses oppositions entre Pavie et les duchés.

De Rothari à Liutprand (636-744)Le roi et la loi

L'accession à la royauté de Rothari ne bouleverse en rien l'attitude du roi lombard à l'égard des duchés, celui-ci cherchant toujours à affirmer sa primauté sur les ducs. Toutefois l'édit de Rothari ${ }^{36}$ en 643 confère au roi une nouvelle dimension, celle d'un législateur et contribue à renforcer sa position face aux ducs. En effet, l'édit de Rothari, complété par presque tous ses successeurs, établit une organisation hiérarchique du royaume, à la tête de laquelle se trouve le roi ${ }^{37}$. Les six premiers articles de cet édit, s'attachent plus ou moins directement à garantir la personne du roi ou son autorité ${ }^{38}$. L'article premier protège la personne du roi contre le régicide. Dans le second article, il est dit que, celui qui tue au nom du roi, ne peut encourir de peine, puisque le cœur du roi se trouve entre les mains de Dieu. Le pouvoir royal prend alors une dimension religieuse qui le place au sommet de la hiérarchie du royaume. Les successeurs de Rothari, Grimoald et Liutprand, qui ont apporté leur contribution à l'œuvre législative, mettent également en avant le lien entre le roi et Dieu derrière cette législation. Ils s'imposent comme législateurs garant de la volonté divine. Ils confirment ainsi le caractère unique de leur position au sein de leur peuple, et plus encore, en ajoutant cette nouvelle aura à leur fonction, se placent au dessus des ducs qui ne peuvent dès lors plus rivaliser avec eux ${ }^{39}$. Cette évolution s'inscrit dans le cadre du renforcement du pouvoir royal et de la centralisation du gouvernement du royaume. Toutefois, les nombreux emprunts faits aux corpus normatifs franc, bavarois et byzantin, laissent penser que les rois lombards cherchaient à imiter leurs voisins immédiats, et par là même à rivaliser avec eux ${ }^{40}$. De fait, cette mutation est à considérer autant à la lumière de l'histoire interne du royaume, qu'à celle d'une royauté lombarde soucieuse d'accroitre son rayonnement face à ses voisins.

Grimoald : l'association du regnum et des duchés du sud

La prise de pouvoir du duc de Bénévent, Grimoald (662-671), en 662 introduit un certain nombre de changements, au premier rang desquels l'association plus étroite des duchés du sud, Spolète et Bénévent, au regnum. Il faut à ce sujet rester prudent quant à la formulation des liens qui unissent le roi à ces duchés du sud, dans la mesure où, ceuxci, n'appartiennent jamais stricto sensu au royaume lombard. Grimoald écarte donc les deux fils d'Aripert (653-661), Godepert et Perctarit, pour s'emparer du pouvoir. Il tue dans un premier temps Godepert, et contraint son frère à la fuite. Cette éviction ne constitue pas à proprement parler une rupture avec la dynastie bavaroise, puisque 
Grimoald épouse une fille d'Aripert ${ }^{41}$, toutefois, celle-ci, a dû susciter des tensions au sein du royaume entre partisans de Perctarit (attachés à la dynastie bavaroise) et partisans de Grimoald. Il convient dans un premier temps de revenir sur les circonstances de l'arrivée de Grimoald à la tête du royaume.

À la mort d'Aripert en 661 , le principe héréditaire semble bien ancré, mais ses modalités encore mal définies. Ainsi, pour la première fois, le royaume est partagé entre les deux fils du roi défunt: Godepert et Perctarit. Le résultat de la double succession semble être une partition du royaume, ou tout au moins une gestion bicéphale; Godepert s'installe à Pavie tandis que Perctarit réside à Milan ${ }^{42}$. Ressurgit alors la vieille capitale d'Agilulf, Milan. Peut-être Godepert était-il l'aîné et pour cette raison obtient Pavie, la capitale traditionnelle depuis Rothari. Ce partage tardif peut, peut-être, traduire des influences franques. Cette partition n'a pas dû se faire pacifiquement, comme en témoigne un diplôme de Perctarit, faisant référence au tempore illo, quando Godepert invasione fecit. Quoi qu'il en soit, Grimoald profite de la situation et probablement des tensions entre les deux frères pour s'emparer du pouvoir. Fait intéressant, il élimine dans un premier temps Godepert et s'empare de Pavie, est-ce à dire que s'emparer de Pavie revient à s'emparer du royaume ? C'est fort probable. Dans un second temps, Perctarit, incapable de lutter contre Grimoald s'enfuit. Les étapes de sa fuite sont assez éclairantes sur les soutiens dont il pouvait bénéficier et les duchés attachés à la dynastie bavaroise. Ainsi, Perctarit part de Pavie, où Grimoald le retenait prisonnier, vers Asti (où restaient des amis de Perctarit demeurés jusque-là rebelles à Grimoald). Perctarit se dirige ensuite au plus vite vers Turin, passe les postesfrontière de l'Italie et arrive en terre franque ${ }^{43}$. On retrouve dans les étapes de cette fuite les cités qu'Agilulf et ses successeurs s'étaient efforcés d'intégrer, notamment par des alliances, et en particulier Asti, qui était le duché qu'avait tenu le grand père de Perctarit, Gundoald. Il restait donc des duchés fidèles à la dynastie bavaroise, et des conflits entre partisans de Grimoald et partisans de Perctarit ont dû avoir lieu, même si les sources n'en font pas directement mention.

17 Le règne de Grimoald est marqué par la volonté d'association plus étroite des duchés du sud au regnum, et par le désir de tisser des solidarités entre le nord et le sud. Il est intéressant de constater qu'immédiatement après avoir été confirmé dans son pouvoir à Pavie, le roi renvoie l'armée bénéventaine, qui l'avait aidé à conquérir le royaume, mais qu'il garde auprès de lui un certain nombre de Bénéventains, à qui il octroie de très vastes possessions ${ }^{44}$. Ce premier geste est destiné à installer dans le regnum un réseau de soutiens favorable au roi. Dans un second temps, Grimoald s'attache à accroître son autorité, comme en témoigne les alliances matrimoniales suscitées entre Spolète, Bénévent et le Frioul.

18 L'action de Grimoald a consisté d'une part à assurer ses positions et celles de son fils Romuald ${ }^{45}$, dans les duchés du sud. C'est à ce titre que Transamund I, ancien comte de Capoue, qui avait assisté Grimoald dans la conquête du pouvoir, est désigné duc de Spolète ${ }^{46}$. De plus, il est contraint d'épouser l'une des filles du roi. Grimoald, dans ce cas précis, place à la tête du duché un fidèle qui l'avait aidé, et renforce le lien de fidélité existant, par un lien de parenté.

19 L'attitude de Grimoald à l'égard du duché de Frioul, s'explique non seulement par ses origines - il est le fils du duc de Frioul Gisulf II (590-610) ${ }^{47}$-, mais aussi par le prestige attaché à ce même duché. Comme je l'ai déjà évoqué, il s'agit du premier duché lombard fondé, ce territoire est en contact avec les Avars et les Byzantins et dispose de 
fait d'une force militaire conséquente. L'adhésion du Frioul au regnum et à la personne du roi constitue par là même un enjeu essentiel. L'importance du duc de Frioul apparaît clairement lors de la révolte du duc Loup en 663. Celui-ci, avait été désigné " régent » du royaume lors d'une expédition de Grimoald en Bénévent ${ }^{48}$. Profitant de l'absence du roi, il se révolte contre Grimoald. Le roi fait alors appel aux Avars ${ }^{49}$ afin de se débarrasser du rebelle, et surtout pour ne pas intervenir lui-même militairement. En effet, Grimoald voulait éviter une guerre civile avec un duché puissant et symbolique. Après l'élimination de Loup, Grimoald contraint la fille du vaincu, Théoderade, à épouser son propre fils Romuald ${ }^{50}$. Par ce geste, il éloigne la fille du duc, la fait entrer dans sa parentèle et désamorce ainsi des velléités de révolte des partisans de Loup, qui aurait pu trouver une légitimité en épousant sa fille. L'importance du puissant duché du Frioul se retrouve également sous le règne de Cunipert (678-700). À la suite de la fuite du duc Rodoald (peut-être un parent installé par Grimoald) devant un usurpateur Ansfrit, le roi intervient et exile ce dernier. Il désigne alors le frère de Rodoald, Ado, non comme duc, mais comme loci servator (lieutenant), autre moyen de minimiser la puissance d'un duché qui semble clé géographiquement pour le royaume et politiquement pour le roi ${ }^{51}$. À partir des règnes de Perctarit et Cunipert, les sources font d'ailleurs de plus en plus souvent référence à la désignation d'autorité des ducs par le roi. Le cas du duc Alahis, est à ce titre particulièrement signifiant, dans la mesure où celui-ci, déjà duc de Trente, est désigné de surcroît duc de Brescia par Cunipert ${ }^{52}$.

L'accession de Grimoald au pouvoir est donc marquée par la volonté du roi de renforcer sa position au nord, mais également de renforcer les solidarités, les liens avec le sud où il était duc. Cette association du nord et du sud peut s'expliquer par les origines de Grimoald. Le retour de Perctarit au pouvoir, marque le retour de la dynastie bavaroise, et, est empreint des mêmes préoccupations : maintenir les acquis de Grimoald, garantir les liens avec les duchés du sud et notamment Bénévent. C'est à ce titre, que la sœur de Cunipert, Wilinga, est donnée en mariage au duc de Bénévent, Grimoald II ${ }^{53}$. Bref, tout au long des $\mathrm{VII}^{\mathrm{e}}$ et $\mathrm{VIII}{ }^{\mathrm{e}}$ siècles, les rois lombards tentent de s'inscrire dans un réseau de parenté efficace, leur conférant à la fois légitimité et soutiens.

Liutprand, stabilité et apogée du royaume

21 L'arrivée de Liutprand marque à nouveau un tournant dans l'histoire du royaume lombard. Il est issu d'une famille de fonctionnaires du palais, implantée à Pavie et restée fidèle à Cunipert et Liutpert, face à Aripert II (703-712). Son père, Ansprand, avait été désigné par Cunipert peu avant sa mort, tuteur de Liutpert, alors mineur. Il exerçait donc la régence du royaume en attendant la majorité du jeune roi, avant d'être renversé, en 703, par le duc de Turin Raginpert et son fils Aripert. Raginpert meurt la même année, et son fils monte sur le trône. Ansprand et Liutprand se réfugient alors chez les Bavarois, qui leur offrent un soutien militaire. À la tête d'une armée de Bavarois, Ansprand et son fils écrasent Aripert en 712. À l'issue de cette bataille Ansprand monte sur le trône, mais meurt la même année. Liutprand lui succède alors. Celui-ci n'est donc ni duc, ni membre apparenté à la famille royale. Ce qu'il ne cherchera d'ailleurs pas à faire, tout au moins pas directement, puisqu'il épouse la fille du duc de Bavière, Guntrud. Or, la modestie des origines de Liutprand est à l'origine de nombreuses tentatives pour se débarrasser de lui ${ }^{54}$, et du soulèvement de certains duchés. Ces duchés rebelles sont parmi les plus puissants et sont attachés à ses prédécesseurs par des mariages comme nous l'avons vu : Spolète, Bénévent et le Frioul. Là encore, il importe de prendre en compte les réseaux de soutiens qui se dessinent de part et d'autre. Ceux hérités de Cunipert et favorables à Liutprand, ceux issus de la 
famille d'Aripert II (Turin notamment, dont le père du roi, Ragimpert, était duc ${ }^{55}$ ). D'autant que dans ce jeu déjà complexe, les Byzantins profitent de la situation fragile du roi pour fomenter des révoltes dans les duchés de Spolète et Bénévent.

Face aux révoltes et aux remises en cause de son autorité, les modalités d'action de Liutprand demeurent les mêmes que celles de ses prédécesseurs. Ainsi, le duc de Frioul, Pemmo, pour s'en être pris au patriarche d'Aquilée contre l'avis du roi, est remplacé par son fils, Ratchis, sur l'ordre de Liutprand ${ }^{56}$. La situation se révèle plus délicate pour les duchés de Spolète et Bénévent. D'une part, ceux-ci ne relèvent pas de l'autorité du roi, puisque n'appartenant pas au regnum, et d'autre part, aucun lien direct ne les relie à la personne du roi. Tout d'abord, le duc de Spolète, Transamund II, aidé des Byzantins, entre en rébellion contre Liutprand ${ }^{57}$. Parallèlement, à la mort du duc de Bénévent, Romuald II, en 731/2, son fils Gisulf II (encore enfant) est menacé par une révolte, conduite par un certain Audelahis ${ }^{58}$. Liutprand intervient alors directement dans ces deux affaires, bien que les deux duchés ne relèvent pas de son autorité. Il est vrai que la trop grande proximité de Spolète avec les Byzantins, de même qu'une relative faiblesse de Bénévent, susceptible de profiter à ces derniers, risquaient de fragiliser considérablement la position du royaume lombard. D'autre part, Romuald II s'était vu attribuer (selon les termes de Paul Diacre) pour femme, Gumperge, la sœur de Liutprand, puis Ranigonde la fille du duc de Brescia ${ }^{59}$. Liutprand est très certainement l'instigateur de ces deux mariages, qui justifient également son intervention pour protéger son neveu, Gisulf. En cela, Liutprand a poursuivi la même politique d'association des duchés du sud au regnum, espérant peut-être susciter des actions conjointes, en tous les cas mieux assurer les frontières avec les Byzantins. De même, après s'être rendu à Bénévent, il place son neveu Grégoire à la tête du duché et se charge de l'éducation de son neveu Gisulf, renforçant par là les liens personnels avec le futur duc de Bénévent. Le roi décide également des mariages des ducs de Bénévent. Il donne à Grégoire Giselperge et à Gisulf une certaine Scauniperge ${ }^{60}$. On peut se demander si les deux femmes n'appartiennent d'ailleurs pas à la même famille, proche de Liutprand. Enfin, à Spolète, il place également un neveu, Agiprand, après avoir chassé Transamund II ${ }^{61}$. Liutprand affirme ainsi la prééminence du roi lombard sur tous les duchés, y compris ceux du sud. Il y désigne le duc et impose les unions. Le népotisme renforce non seulement l'autorité du roi, mais pérennise également sa position à la tête du royaume.

L'intégration des duchés lombards dans le regnum, est avant tout l'insertion du roi dans un réseau de parenté efficace. D'Authari à Liutprand, les rois lombards tentent d'imposer leurs candidats à la tête des duchés. Leur motivation est double, garantir d'une part l'intégrité des frontières du royaume face à des voisins belliqueux, d'autre part asseoir leur autorité au sein même du royaume. Cette stratégie se heurte souvent à des ducs dont le prestige concurrence celui du roi, et à des politiques locales de transmission du pouvoir qui se mettent progressivement en place au cours des $\mathrm{VII}^{\mathrm{e}}$ et $\mathrm{VIII}^{\mathrm{e}}$ siècles. Le problème des fondements de la légitimité du pouvoir des rois lombards est donc un enjeu essentiel dans la compétition qui les oppose aux ducs. L'appartenance ou le rattachement à une puissante lignée, le renforcement des prérogatives royales et la «sacralisation" du pouvoir contribuent à accroître le rayonnement du roi tant à l'intérieur du regnum, que face à ses voisins. Néanmoins, la place du roi demeure fragile, dans la mesure où le principe héréditaire s'impose difficilement. En témoignent les multiples révoltes qui alimentent l'histoire du royaume. Ainsi, du vi au vIII siècle, 
l'histoire de la royauté lombarde se confond largement avec celle du conflit qui oppose la volonté centralisatrice et unificatrice des rois à la fragmentation du pouvoir et de l'espace entretenue par les ducs.

\section{NOTES}

1. P. DiACRE, Historia Langobardorum (désormais abrégé en : HL), éd. L. BETHMANN et G. WAITZ, MGH SS RL, Hanovre, 1878, p. 12-187. HL III, 16. Traduction F. BOUGARD, Histoire des Lombards, Brepols, 1994, p. 64-65.

2. P. DeLOGU, «Il regno longobardo », Storia d'Italia, vol. 1 : Longobardi e Bizantini, éd. P. Delogu, A. Guillou et G. ORTAlli, Turin, 1980, p. 17-24. J. JaRnut, Geschichte der Langobarden, Urban 339, Stuttgart, 1982, p. 37-39. W. PoHL, « The Empire and the Lombards : treaties and negotiations in the sixth centuries ", dans Kingdoms of the Empire. The integration of Barbarians in Late Antiquity, ed. W. POHL, The Transformation of the Roman World I, Leiden-New York-Köln, 1997, p. 75-134.

3. S. GASPARRI, I duchi Longobardi, Rome, 1978, Studi Storici 109, p. 25-32. S. GASPARRI, « Pavia longobarda », Storia di Pavia, vol. 2, L'Alto Medioevo, Pavie, 1987, p. 19-65.

4. Sur la question du regnum, J. JARNUT, « Gens, rex and regnum of the Lombards ", dans Regna and Gentes, the relationship between Late Antique and Early Medieval peoples and kingdoms in the transformation of the Roman World, éd. H.-W. GOETZ, J. JARNUT and W. POHL, Brill, 2003. p. 409-427.

5. H. FRÖHLICH, Studien zur langobardischen Thronfolge. Von den Anfängen bis zur Eroberung des italienischen Reiches durch Karl den Grossen (774), 2 vols, Tübingen, 1980.

6. HL II, 31. Les Lombards élisent à l'unanimité Cleph, de leur plus haute noblesse, comme roi à Pavie.

7. FRÉDÉGAIRE, Chronicon, ed. B. KRUSCH, MGH SSrM 2, Hanovre, 1888, IV, 45, p. 143.

8. HL III, 9.

9. HL III, 17.

10. Die Bajuwaren. Von Severin bis Tassilo 488-788, éd. H. DANNHEIMER et H. DoPSCH, Rosenheim-Salzburg, 1988. J. JARNUT, « Beiträge zu den fränkish-bayerishlangobardischen Beziehungen im 7. und 8. Jahrhundert », Zeitschrift für bayerische Landesgeschichte 39, 1976, p. 331-352.

11. HL III, 30 et 35. FRÉDÉGAIRE, Chronicon, op. cit., IV, 13, p. 127.

12. HL IV, 41. FréDÉGAIRE, Chronicon, op. cit., IV, 50, p. 145. Voir également la prosopographie effectuée par S. GASPARRI, I duchi Longobardi, op. cit., p. 51-52.

13. HL III, 29 et 31.

14. A. SETTIA, « Le frontiere del regno Italico nei secoli VI-IX : l'organizzazione della difesa ", Frontières et peuplement dans le monde méditerranéen au Moyen Âge, Castrum 4 , Rome-Madrid, 1992, p. 201-209.

15. HL IV, 42. FRÉDÉGAIRE, Chronicon, op. cit., IV, 70, p. 156.

16. HL III, 18 et 19 au sujet de Droctulf. HL IV, 8 au sujet de Maurice. S. GASPARRI, I duchi Longobardi, op. cit., p. 54 et 59. 
17. HL IV, 13.

18. HL II, 28. Paul Diacre évoque Vérone comme le lieu de résidence du roi Alboin, ainsi que le lieu de son inhumation.

19. G. P. BRogiolo, «Capitali e residenze regie nell'Italia longobarda », Sedes Regiae (ann. 400-800), éd. G. RIPOLL and J. M. GuRT with A. ChAVARRIA, Barcelone, 2000, p. 135-162.

20. HL II, 32. Paul Diacre mentionne après la mort de Cleph, la présence d'un certain Zaban, duc de Pavie.

21. G. WoLF, « Königin Theodelinde als Heils und Legitimitätsträgerin und die langobardisch-bayerisch-fränkischen Beziehungen um 600 », Zeitschrift der SavignyStifung für Rechtsgeschichte, Germ. Abt., Bd. 106, 1989, p. 284-290.

22. FrédÉGAIRE, Chronicon, op. cit., IV, 50, p. 145.

23. FRÉDÉGAIRE, Chronicon, op. cit., IV, 70, p. 156. S. Gasparri, I duchi Longobardi, op. cit., p. 43.

24. HL IV 47.

25. HL I, 21. J. JARNUT, « Zur Frühgeschichte der Langobarden », Studi Medievali 3, 1983, p. 1-16.

26. Au sujet des Agilolfing J. JARNUT, Agilolfingerstudien Untersuchungen zur Geschichte einer adlingen Familie im 6 und 7 Jahrhundert, Stuttgart, 1986.

27. J. LECLERCQ, "Rôle et pouvoir des épouses au Moyen Âge », La Femme au Moyen Âge, ed. M. Rouche et J. Heucuin, Maubeuge, 1990, p. 87-97. Sur le rôle de la reine :

P. STAFFORD, Queens, Concubins and Dowagers, Athens-Georgie, 1981.

28. HL IV, 40. FRÉDÉGAIRE, Chronicon, op. cit., IV, 34, p. 134.

29. HL III, 30.

30. S. GASPARRI, I duchi Longobardi, op. cit., p. 16.

31. HL III, 10. Le Duc Evin de Trente épouse la seconde fille de Garibald.

32. HL II, 32.

33. HL III, 9.

34. HL III, 27.

35. HL IV, 1.

36. Edictus Rothari, ed. F. BluHME, MGH LL, Hanovre, 1888, p. 1-90.

37. J. JARNUT, " Gens, rex and regnum of the Lombards », op. cit., p. 419.

38. Edictus Rothari, art. 1 à 6, p. 2-3.

39. D. HARRISON, The Early State and the Towns. Forms of Integration in Lombard Italy, AD 568-774, Lund Studies in International History 29, Lund, 1993, p. 105-9. ID., « Political rhetoric and political ideology in Lombard Italy ", Strategies of Distinction. The construction of Ethnic Communities, 300-800, ed. W. POHL et H. REIMITZ (The Transformation of the Roman World 2), Leiden-New York-Köln, 1998, p. 241-54.

40. C. AzZARA, S. GASPARRI, Le leggi dei langobardi : storia, memoria, diritto di un popolo germanico, Milan, 1992, p. XXII-XXXIV.

41. HL V, 1. S. GASPARRI, I duchi Longobardi, op. cit., p. 88-89.

42. HL IV, 51.

43. HL V, 2 .

44. HL V, 1 .

45. S. GASPARRI, I duchi Longobardi, op. cit., p. 89-90.

46. HL IV, 51 et V, 16. S. GASPARRI, I duchi Longobardi, op. cit., p. 76.

47. S. GASPARRI, I duchi Longobardi, op. cit., p. 66. 
48. $H L \mathrm{~V}, 17$. « C'est à Loup que Grimoald confia son palais quand il partit pour

Bénévent ». S. GASPARRI, I duchi Longobardi, op. cit., p. 67-68.

49. HL V, 19.

50. HL V, 25.

51. HL VI, 3

52. HL V, 36. S. GASPARRI, I duchi Longobardi, op. cit., p. 24.

53. HL VI, 2.

54. HL VI, 38. Paul Diacre évoque notamment un parent, un certain Rothari, qui voulut le supprimer.

55. HL VI, 18.

56. HL VI, 51.

57. HL VI, 55 et 56.

58. Voir à ce sujet l'hypothèse de S. GASPARRI, I duchi Longobardi, op. cit., p. 92-93.

59. HL VI, 50.

60. HL VI, 55.

61. HL VI, 57.

\section{RÉSUMÉS}

En 584, le territoire contrôlé par les Lombards est éclaté en de multiples duchés. Ceux-ci, autonomes, sans réelle cohésion entre eux risquaient d'être absorbés par leurs puissants voisins francs et byzantins. C'est dans ce contexte qu'il faut envisager la restauration de la royauté lombarde. Tout au long des $\mathrm{VI}^{\mathrm{e}}, \mathrm{VII}^{\mathrm{e}}$ et $\mathrm{VIII}^{\mathrm{e}}$ siècles les rois lombards se heurtent aux velléités autonomistes de leurs ducs, qui mettent en péril la cohésion du royaume. Ces derniers sont en perpétuelle compétition avec le pouvoir royal, dont ils refusent les tentatives de centralisation. Les rois lombards d'Authari à Liutprand tentent d'asseoir leur autorité sur le regnum, mais aussi d'établir leur primauté à la tête de celui-ci. Cette entreprise passe notamment par la prise de contrôle progressive des duchés les plus turbulents, en y installant des membres de leur parentèle ou des fidèles. Cependant, cette stratégie conduit à de nombreuses révoltes, qui fragilisent le pouvoir royal. Cette étude propose donc d'envisager la construction du royaume lombard sous l'angle de la lutte entre les duchés et le pouvoir royal, en mettant en lumière les réseaux de soutiens ou de contestations de la royauté, et la manière dont celle-ci parvient s'imposer face aux duchés.

The Kingdom of Lombardy and the Duchies: Forms and Methods of a Progressive Integration. In 584 the territory controlled by the Lombards was split up into several autonomous duchies. These lacked any real cohesion and were at risk of being absorbed by their powerful Frankish or Byzantine neighbours. It is in this context that the restoration of the Lombard royalty must be envisaged. Throughout the 6th, 7th and 8th centuries the Lombard kings struggled with the dukes desire for independence, who thus put the kingdom in peril. The latter were in perpetual conflict with the royal power, refusing their attempts at centralisation. The Lombard kings from Authari to Liutprand tried to base their authority on the regnum, but also to establish their position at its head. In particular they effected this enterprise through the progressive control of the most turbulent of the duchies by installing at their head the members of their family or their devoted 
followers. However, this strategy led to numerous rebellions that weakened the royal power. This study proposes therefore to view the construction of the Lombard kingdom from the point of view of the struggle between the duchies and the royal power, highlighting the network of support or contestation of the kingdom and the way in which the latter managed to impose itself in the face of the duchies.

INDEX

Mots-clés : duché, regnum, continuité dynastique, Bénévent, Agilolfing, Théodelinde, parenté efficace

Keywords : duchy, dynastic continuity, Benevento, Agilofing, Theodelind, blood relationship

\section{AUTEUR}

YVES-MARY VERHOEVE

Université Lille III-Charles-de-Gaulle, UMR 8529 IRHIS, BP 60149, 59653 Villeneuved'Ascq Cedex 